\title{
Effect of Mitchell Relaxation technique and general mobility exercises on post partum eclampsia, stress and stress induced convulsions- A Case Study.
}

\author{
Paras Joshi, \\ Physiotherapist, PDU Government Hosptial, Rajkot, India
}

\begin{abstract}
: post partum stress and hyper tension is common disorder in women, however moderate to severe stress and depression may lead to convulsions. A lady having a similar complaint was being treated with Mitchell relaxation technique, deep breathing exercises and other mobility exercises for a period of 10 weeks. All the investigations like MRI, ECG, Blood Reports were found normal or near normal. There was no specific clinical correlation found between any of the investigation report to eclampsia and seizures except level of stress. There was good reduction found on depression, anxiety and stress. Post partum seizures have reduced to zero. Hypertension was also able to decline to normal range. From the case it can be concluded that Mitchell relaxation techniques combined with other stress reliving exercises can be use to treat post partum eclampsia and related stress. Stress induced convulsion may be treated with such exercises.
\end{abstract}

Key words: anxiety, depression, mitchell relaxation technique, post eclampsia, stess

\section{Introduction}

The birth of a child is one of the most intense and emotional experiences in a woman's life and results in extremes in the perception of this event. Both traumatically experienced childbirth and stressful events in the individual life story of a woman can result significant psychological impairments post partum. Depending on objective and subjective factors, childbirth can act as a significant stressor and trigger for post-traumatic stress disorder (PTSD), a mental illness which affects a disproportionately high number of women in childbearing age, with a lifetime prevalence of $10.4-13.8 \%{ }^{1}$

Postpartum depression (PPD), also called postnatal depression, is a type of clinical depression which can affect women, and less frequently men, typically after childbirth. Studies report prevalence rates among women from $5 \%$ to $25 \%$.Although a number of risk factors have been identified, the causes of PPD are not well understood. Many women recover with a treatment consisting of a support group or counseling. ${ }^{2,3}$

Post partum convulsions are very rarely to occur; and remained diagnostic enigma. ${ }^{4}$

A study shows that there were 54 cases of late postpartum eclampsia among a total of 334 cases of eclampsia during the study period. Late postpartum eclampsia constituted $56 \%$ of total postpartum eclampsia and $16 \%$ of all cases of eclampsia. Convulsions began from postpartum days 3-23 (mean 6). Thirty women $(56 \%)$ had been identified as preeclampsia before their convulsions. A history of either severe headache or visual disturbances before convulsion was elicited in $83 \%$ of the patients. Only eight of 62 patients with late postpartum seizures had identifiable etiologies other than eclampsia. ${ }^{5}$

Exact clinical Diagnosis of present given case was remained unclear after detailed clinical evaluation; however retrospective (for prenatal) stress and anxiety, postnatal stress and anxiety, pre and post eclampsia thought to have stress induced seizures in this patient. Aim of this study is to check the effects of Mitchell relaxation technique and other stress reducing exercises on depression, anxiety and stress in post partum women having post eclampsia and convulsions.

\section{Case Report}

A 24 year old female came with complaint of restlessness and seizures to our physiotherapy department, $6^{\text {th }}$ day post delivery.

\section{II.I Prenatal history.}

Lady was found apparently normal till the 32 weeks of gestation. There was no significant complaint found from lady. High blood pressure was detected first time during routine pre natal check up $(140 / 90 \mathrm{~mm} \mathrm{Hg})$. All the other reports related to fetus and mother was absolutely normal. Summary of blood pressure recorded at various intervals during prenatal phase is listed in table no. From the $32^{\text {nd }}$ week of gestation lady was advised to take nifidepine $5 \mathrm{mg}$ daily by the consultant gynecologist. Lady had taken it as per advice till delivery. Apart from ankle and hand swelling there was absolutely no other complaint from lady. There was no episode of convulsion during prenatal phase. 


\section{II.II Perinatal History.}

She was going to be a fresh mother at $39^{\text {th }}$ weeks of gestation. Labor pain had remained for around 6 to 7 hours. Lady delivered a baby boy with normal full term vaginal delivery. Episiotomy and vacuum cup was used to deliver a baby. Baby was positioned normal and there was no knotted umbilical cord around the neck. There were no signs of infection in baby and mother at the time of delivery. Blood pressure was found normal during the delivery.

\section{II.III Post natal history.}

Lady was found absolutely normal till the $4^{\text {th }}$ day post delivery; on the same late night lady has experienced convulsions for about 6 minutes. On $5^{\text {th }}$ day post delivery, in early morning lady had experienced another episodes of convulsion which last for about 10 minutes. Lady was fully conscious throughout the episodes of convulsion, but she was not oriented to place, person and time for about 15 to 20 minutes. Immediately she was admitted to one private hospital. Blood pressure was found at that time was $150 / 106 \mathrm{~mm}$ of Hg. Patient was given Inj. Labetol (1 amp)+ 100cc IV, Inj. Phenargan (1 amp), Tab Methyl Dopa (250mg) 3 stat, Tab. Nicardia (10mg) 1-0-1 during 24 hours of hospitalization. She responded very well to medications and discharged on $6^{\text {th }}$ day post delivery. On $6^{\text {th }}$ day post delivery general stress reliving exercises along with Mitchell relaxation techniques have been taught to patient. Patient was regularly taking medicines M- dopa and Nicardia as per advice. Patient started slowly weaning the medications. Blood pressure during the weaning time was found normal. She completely stopped the medications on $13^{\text {th }}$ post delivery day as per advice by consultant. Blood pressure was found normal till the morning $15^{\text {th }}$ post delivery day. On the same late night patient has experienced convulsions again for 3 minutes. Duration as well as intensity of convulsions was less than previous according to care givers. Blood pressure immediately after the convulsions found higher (146/100 $\mathrm{mm} \mathrm{Hg}$ ). She experienced convulsion $4^{\text {th }}$ time for about 2 and half minutes on the $16^{\text {th }}$ day post delivery. Again the same medications started by a consultant gynecologist, later on only nicardia were being given for twice a day. She has been referred to neuro physician. She has been advised for MRI and EEG reports. Both the reports were found normal with no signs of any infections and abnormal EEG activities. Physician started Levetiracetam $500 \mathrm{mg}$ as anticonvulsive medicine. From the same day lady had started stress reliving exercise program.

\section{Interventions}

Retrospective DASS (Depression, Anxiety and Stress Scale) was taken at $36^{\text {th }}$ week of gestation, $6^{\text {th }}$ day post delivery, 1 month post delivery, and 3 months post delivery. Blood pressure was continuously monitored through out the treatment sessions. It may be noted that drugs to relive blood pressure was totally weaned off by 1 month post delivery. However anticonvulsive drugs were being taken regularly till the end of this study.

Treatment was started at the $6^{\text {th }}$ day post delivery. Various Exercise protocol had been introduced to reduce the Depression, Anxiety and Stress focused mentally and physically.

Summary is mentioned in the Table no 1.

Mitchell Relaxation Technique ${ }^{6}$ : (Fig. 1A)

It contents easy breathing and slow movement of almost each of the joint while lying flat on back with no distraction. Example for shoulder joint is given below.

SLIDE your hands along the floor down towards your knees, pulling your shoulders down. Stop pushing and let go. .............Repeat

THINK about the movement.

FEEL the new position of the shoulder joints, feel them loose and down.

10 repetitions for each joint and body was given.

Progressive Muscle Relaxation Technique:

It starts from Right foot and ends in face.

Subject is instructed to contract particular part by making it tight for 10 seconds and then relax. And then move to next body part in given sequence. Example to start with is given below.

Loosen your clothing, take off your shoes, and get comfortable. Take a few minutes to relax, breathing in and out in slow, deep breaths. When you're relaxed and ready to start, shift your attention to your right foot. Take a moment to focus on the way it feels. Slowly tense the muscles in your right foot, squeezing as tightly as you can. Hold for a count of 10. Relax your right foot. Focus on the tension flowing away and the way your foot feels as it becomes limp and loose. Stay in this relaxed state for a moment, breathing deeply and slowly. When you're ready, shift your attention to your left foot. Repeat the Same. 
Diaphragmatic Breathing Exercise ${ }^{7}$ : (Fig. 2)

Subject was asked to sit in semi fowler's position (gravity assisted position for Diaphragm), and instructed in the following manner

Be relaxed. Put your hand over your abdomen (epigastric area), take deep breath in through nose and breathe out through mouth gently. When you are breathing in, your stomach should rise up and while breathing out it should come down.

During pregnancy excursion of diaphragm was hampered by uterus. And breathing might have been altered so in order to reduce the same and to reduce the stress this particular exercise have been taught to the patient.

Walking for 15 minutes at least:

Patient was advised to walk at comfortable pace for at least 15 minutes. Patient was walking for 30 minutes after 45 days post delivery.

General Mobility Exercises to correct the Posture :

Studies suggest that altered posture and pain may lead to stress and depression. A pregnant woman is likely to get such altered posture. After detail evaluation patient was given following exercises in order to correct her posture

1. Posterior pelvic tilt exercises against wall to correct excessive anterior pelvic tilt and lumbar lordosis (Fig. 1B)

2. Static and dynamic abdominal exercises along with pelvic floor exercises to correct the weakness of abdominal and core muscles

3. Correction of kyphotic posture

4. Pectoralis major stretching to correct the tight pectoral group

5. Scapular retractor muscle strengthening to strengthen weak rhomboids and middle trapezius

6. Upper Trapezius muscles exercises

7. Strengthening for cranio cervical flexors to correct the pocking chin.

Correct techniques for baby care and feeding:

1. Nappy changing on height to avoid stress on back

2. Baby massage technique on lap with back support

3. Breast feeding technique: keeping the baby as close to chest as possible, with full upper back support and shoulders drawn posterior.

Psychological counseling:

Patient was given psychological counseling every 15 days regarding enjoying mother hood and other psychological factors by psychologist

\section{Results:}

Depression was able to decline on scale from 13 (mild) to 10 (mild) at the 3 month post delivery. Anxiety was able to decline on scale from 15 (severe) to 4 (normal) at the 3 month post delivery. Stress was able to decline on scale from 27(severe) to 9 (normal) at the 3 month post delivery. Blood pressure was maintained around $110 / 72 \mathrm{~mm} \mathrm{Hg}$ till the end of treatment without antihypertensive drugs from $1^{\text {st }}$ month post delivery to $3^{\text {rd }}$ month post delivery. There was no convulsion episode found after $15^{\text {th }}$ day post delivery till the end of the treatment. (Table. 2, Graph 1) (Anticonvulsive drug was being taken as advised by neuro physician.)

\section{Tables, Graphs and Figures.}

Table 1. Exercise protocol: Summary

\begin{tabular}{|l|l|l|}
\hline 1. & Mitchell Relaxation techniques (30 Minutes) & Daily \\
\hline 2. & Progressive Muscles Relaxation Technique (10 Minutes) & Three days/ Week \\
\hline 3. & Diaphragmatic Breathing Exercises (5 Minutes or 10 to 12 repetitions) & Daily \\
\hline 4. & Walking for 15 Minutes at least & 4 times a week \\
\hline 5. & $\begin{array}{l}\text { General Mobility exercises focused on to correct the posture (10 } \\
\text { Minutes) }\end{array}$ & Three Days/ Week \\
\hline 6. & Correct techniques for baby care and feeding & Two days/ Week \\
\hline 7. & Psychological counseling & Every fortnight \\
\hline
\end{tabular}


Effect of Mitchell Relaxation technique and general mobility exercises on post partum eclampsia,

Table 2. DASS, Blood pressure and Episodes of Seizures during Prenatal and Post natal duration.

\begin{tabular}{|l|l|l|l|l|}
\hline Duration/Variables & $\begin{array}{l}36^{\text {th }} \text { weeks of } \\
\text { gestations }\end{array}$ & $6^{\text {th }}$ day post delivery & 1 month post delivery & $\begin{array}{l}3 \text { months post } \\
\text { delivery }\end{array}$ \\
\hline Depression & 13 (Mild) & 20 (Moderate) & 12 (Mild) & 10 (Mild) \\
\hline Anxiety & 15 (Severe) & 17 (Severe) & 9 (Mild) & 4 (Normal) \\
\hline Stress & 27 (Severe) & 29 (Severe) & 14 (Normal) & 9 (Normal) \\
\hline Total & 55 & 66 & 35 & 23 \\
\hline Blood pressure in mm Hg & $140 / 90$ & $132 / 86$ & $110 / 70$ & $110 / 72$ \\
\hline Episodes of seizures till & 0 & 2 & 2 & 0 \\
\hline
\end{tabular}

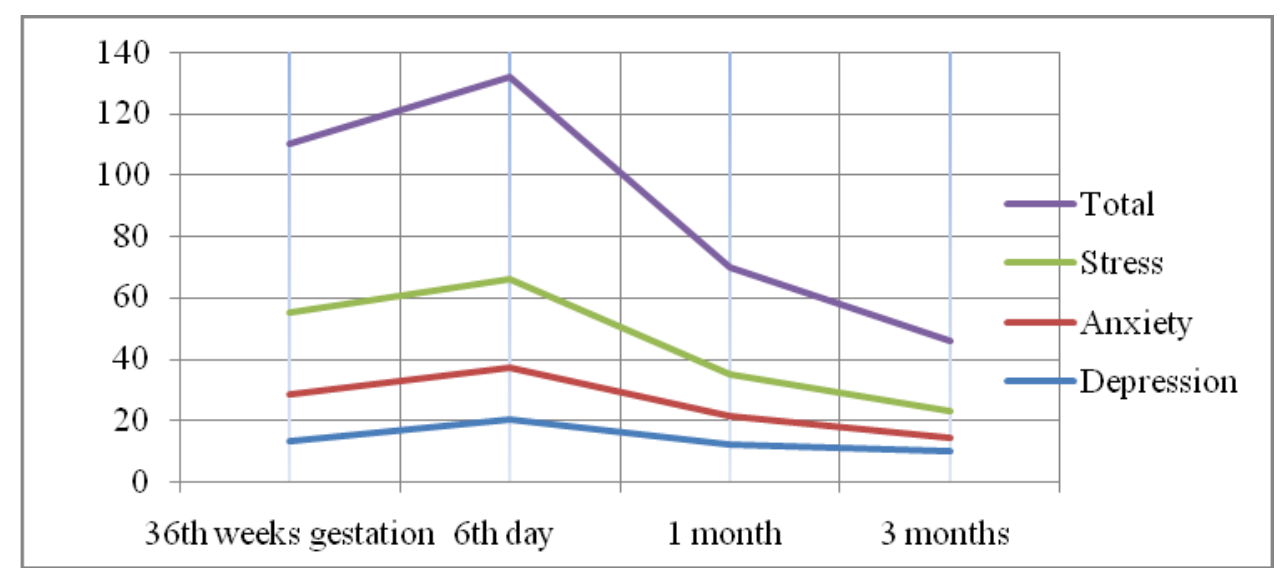

Graph 1. DASS at different phases of prenatal and post natal duration.

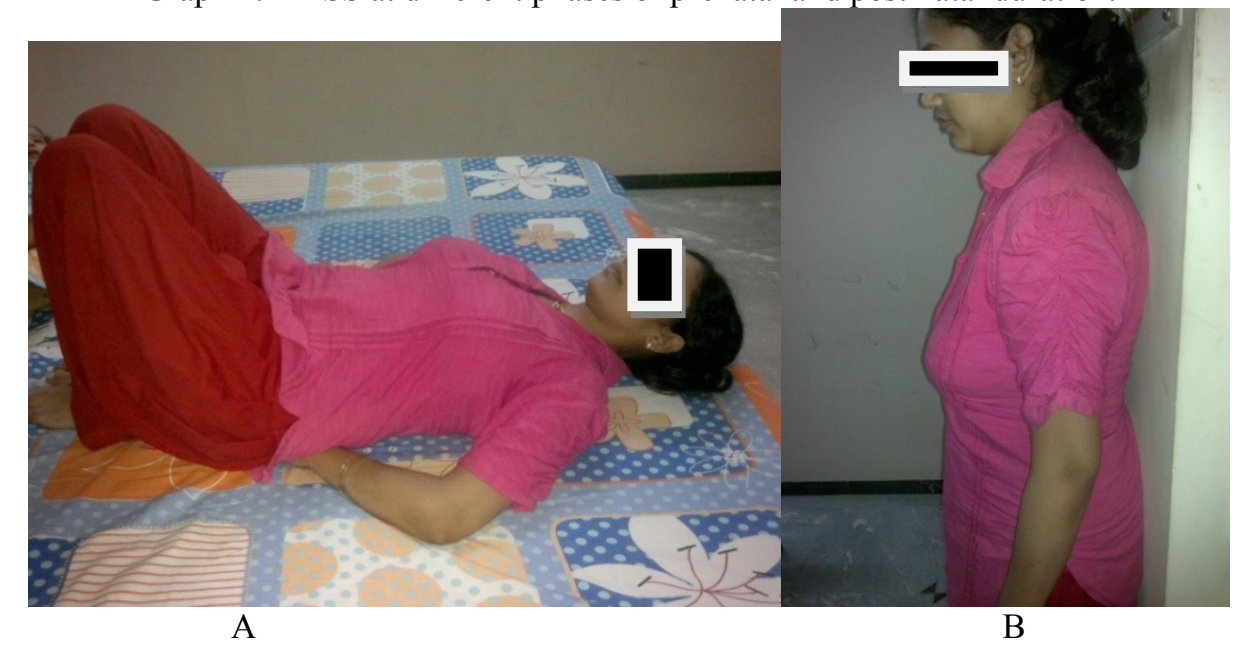

Figure 1. Patient performing part of A. mitchell exercises and B. posterior pelvic tilt exercises

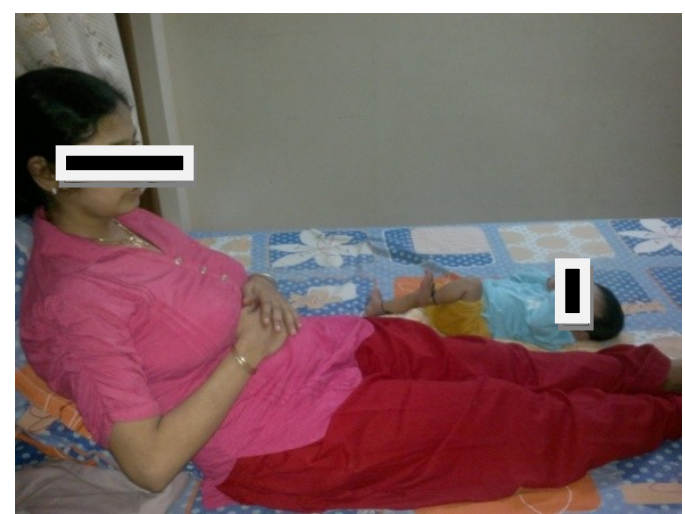

Figure 2. patient performing diaphragmatic breathing exercise. 


\section{Conclusion}

Mitchell relaxation techniques combined with other stress reliving exercises can be effective in treating post partum Stress, Depression and anxiety along with post partum eclampsia. It may be helpful to overcome stress induced convulsions.

It has been observed that altered posture after the pregnancy can cause mental stress along with pain. So posture correction exercises have been purposefully added to this patient.

Diaphragmatic excursion may have been adversely hampered due to uterus size. There for Deep breathing exercise focusing on diaphragmatic excursion have been added to facilitate diaphragm excursion and ease breath.

Baby care is one of the most difficult thing mother has to deal with after delivery; and eventually lead to bad posture due to bad postural habits like forward bending and keeping the shoulder forward. Even increased breast weight can have the faulty kyphotic posture. So proper baby care advice with correct posture have been taught to this patients. Neuro physician, Gynecologist and a consultant Psychologist were active rehabilitative team members for this patient's rehabilitation.

Levetiracetam drug is having a side effect of mood swing and depression; therefore probably we dint get much improvement in patient's depression scale. It may be noted that this drug was on till the end of our treatment so we were not able to correlate the effect of treatment to episodes of convulsion. Stress and hypertension were thought to be having the factors which lead to convulsions in this patient which can be effectively managed without antihypertensive drugs. According to physiology, blood pressure tend to decline after $6^{\text {th }}$ post delivery but here in this patient it was not declined.

Similar study is required with larger sample size.

\section{References}

[1] Yule W.Posttraumatic stress disorder in the general population and in children. J Clin Psychiatry 2001; 62 (Suppl.17): 23-28

[2] Paulson, James F. (2010). "Focusing on depression in expectant and new fathers: prenatal and postpartum depression not limited to mothers" Psychiatry Times 27 (2).

[3] Cox JL, Holden JM, Sagovsky R (June 1987). "Detection of postnatal depression. Development of the 10 -item Edinburgh Postnatal Depression Scale". Br J Psychiatry 150 (6): 782-6.

[4] Anita Dutta, Toni Tonkin, and Wolf Gelman. "Post partum convulstions- a diagnostic enigma" J R Soc Med. 2006 April; 99(4): 203204.

[5] Lubarsky SL, Barton JR, Friedman SA, Nasreddine S, Ramadan MK, Sibai BM. "Late post partum eclampsia revisited". Obstet Gynecol. 1994 Apr;83(4):502-5.

[6] Patricia A Downie, general medical and surgical conditions for physiotherapist edi. $2^{\text {nd }}$ (London, wolf publishing 1993)111-132

[7] Carolyn Kisner, Therapeutic Exercise foundation and techniques edi. $4^{\text {th }}$ (Philadelphia, F.A davis company 2002)750-752.

[8] M Dena Gardinar, The principles of exercise therapy edi. $4^{\text {th }}$ (London, bailliere tindall 2000) 245-255. 